\title{
Study on Parallel Beam Splitting Polarizer with Matrix Method
}

\author{
Yuqing Xie ${ }^{1, a}$, Junjie Wang ${ }^{1, b}$ and Dong Xue ${ }^{1,2, c^{*}}$ \\ ${ }^{1}$ School of Opto-Electronic Engineering, Zaozhuang University, Zaozhuang277160, China \\ ${ }^{2}$ Zaozhuang Engineering Research Center of Terahertz, Zaozhuang 277160, China \\ a Harrison.xie@outlook.com, b 458692589@qq.com, c fiberlaser@126.com
}

Keywords: Parallel beam splitter; Polarizer; Matrix

\begin{abstract}
Jones matrix is a useful method which is widely used in optical research. But there is no existing such matrix for the parallel beam splitting polarizer, because it changes both the direction and polarization of incident light. This document explains and demonstrates how to treat a parallel beam splitting polarizer with matrix method. A block diagonal matrix is designed, in which the top left block is a 3-order transform matrix dealing with the geometrical light propagation, the bottom right block is a 2-order Jones matrix dealing with the polarization. As an application, we use this method to deal with the high efficiency polarizer. The theoretical deduce is coincident with experimental results.
\end{abstract}

\section{Introduction}

The matrix, as a mathematical tool, has penetrated much of optics field and created a system of its own[1-3]. When dealing with geometrical optics problems, we can use the transformation matrix; when dealing with polarization related problems, we can take advantage of Jones matrix; but for dealing with parallel beam splitter, which has both light changes and polarization issues, some matrix methods are helpless. In this paper, a new approach is adopted to combine the two matrix methods to deal with the above problems.

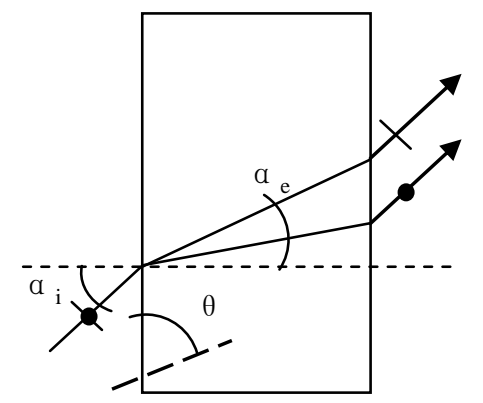

Figure 1. The structure of parallel beam splitter

\section{Theorem}

\section{The Splitting Features of Parallel Beam Splitter}

Parallel is a kind of polarizing beam splitting prism, which is often used in laser applications technology [4,5], its structure and the split optical path as shown in Fig. 1: The optical axis of the crystal and incident plane into a certain angle $\phi$, the incident light is located in the main cross section (the incident light, the refracted light and the optical axis are located in the paper). The vibration directions of two plane polarized split light are perpendicular to each other, but the beam splitting angle is zero, that is, two beams parallel output.

When using the matrix to study geometrical optics, it is necessary to calculate the transformation matrix of optical devices. Parallel beam splitter for o light and e light transformation is different, this is mainly due to the e light does not comply with the law of conventional refraction, which can be clearly seen from Fig.1, so we should discuss separately. For o light, a parallel beam splitter is equivalent to an optical parallel plate, whose transformation matrix is [6,7] 


$$
\mathrm{L}_{0}=\left(\begin{array}{cc}
1 & \frac{L}{n_{o}} \\
0 & 1
\end{array}\right)
$$

Where, $\mathrm{L}$ is the thickness of the parallel beam splitter, and $\mathrm{n}_{\mathrm{o}}$ is the refractive index of the o light.

For e light, according to the crystal optics theory, if the angle between the crystal axis and the incident interface is $\varphi$, and the incident angle is $\alpha_{\mathrm{i}}$, then refraction angle is given by the following formula:

$$
\tan \alpha_{e}=\frac{2\left(n_{o}^{2} \cos ^{2} \theta+n_{e}^{2} \sin ^{2} \theta\right)}{\left(n_{e}^{2}-n_{o}^{2}\right) \sin 2 \theta+2 n_{o} n_{e} \sqrt{\frac{n_{o}^{2} \cos ^{2} \theta+n_{e}^{2} \sin ^{2} \theta}{\sin ^{2} \alpha_{i}}-1}}
$$

When light is incident vertically $\left(a_{i}=0\right)$ :

$$
\tan \alpha_{e}=\left(\frac{n_{o}^{2}}{n_{i}^{2}}-1\right) \frac{\cot \theta}{1+\frac{n_{o}^{2}}{n_{i}^{2}} \cot ^{2} \theta}
$$

The $\alpha_{\mathrm{e}}$ is also called the discrete angle of light e.

Normally we use $\theta$ to indicate the angle between the light and the horizontal axis. The distance from the incident (outgoing) point to the axis is represented by $\mathrm{r}$. When the light is incident perpendicularly, the relation of light parameter between the incident surface and outgoing surface is $\theta_{2}=\theta_{1}=\alpha_{\mathrm{i}}$, $r_{2}=r_{1}+L \tan \alpha_{\mathrm{e}}$.For the convenience of computation, this relation can be written in the form of matrix:

$$
\left(\begin{array}{c}
r_{2} \\
\theta_{2} \\
1
\end{array}\right)=\left(\begin{array}{lll}
1 & 0 & L^{\prime} \\
0 & 1 & 0 \\
0 & 0 & 1
\end{array}\right)\left(\begin{array}{c}
r_{1} \\
\theta_{1} \\
1
\end{array}\right)
$$

Where $L^{\prime}=L \tan a_{\mathrm{e}}$

\section{The Polarized Features of Parallel Beam Splitter}

A parallel beam polarizer is equivalent to two vertical polarizers placed side by side, and the direction of the outgoing light vibration is perpendicular to each other. For horizontal and vertical polarizer, the Jones matrix is respectively $[6,8]$ :

$$
\mathbf{J}_{/ /}=\left(\begin{array}{ll}
1 & 0 \\
0 & 0
\end{array}\right), \quad \mathbf{J}_{\perp}=\left(\begin{array}{ll}
0 & 0 \\
0 & 1
\end{array}\right)
$$

\section{The Block Diagonal Matrix of Parallel Beam Splitter}

In order to reflect the feature of beam splitting and polarization of parallel beam splitter, the incident and outgoing beams are written as column vectors:

$$
\mathrm{A}=\left(\begin{array}{lllll}
r & \theta & 1 & x & y
\end{array}\right)^{T}
$$

$\mathrm{T}$ represents the transpose matrix, $\mathrm{r}$ represents the distance from the principal axis, ${ }^{\theta}$ represents the angle between the light and the principal axis, and $\mathrm{x}$ represents the polarization component in the direction perpendicular to the paper, and y represents the polarization component in the direction parallel to the paper.

For incident components with direction perpendicular to the paper, the parallel beam splitter is equivalent to a parallel glass plate. Compare with Eq. 4 and Eq. 6, the block diagonal matrix is:

$$
\mathbf{M}_{\perp}=\left(\begin{array}{ccccc}
1 & L^{\prime \prime} & 0 & 0 & 0 \\
0 & 1 & 0 & 0 & 0 \\
0 & 0 & 1 & 0 & 0 \\
0 & 0 & 0 & 1 & 0 \\
0 & 0 & 0 & 0 & 0
\end{array}\right)
$$


For incident components with direction parallel to the paper, the light will deviate from its wave normal direction. Compare with Eq. 1 and Eq. 5 , the block diagonal matrix is:

$$
\mathbf{M}_{/ /}=\left(\begin{array}{lllll}
1 & 0 & L & 0 & 0 \\
0 & 1 & 0 & 0 & 0 \\
0 & 0 & 1 & 0 & 0 \\
0 & 0 & 0 & 0 & 0 \\
0 & 0 & 0 & 0 & 1
\end{array}\right)
$$

Where $\mathrm{L}^{\prime \prime}=\mathrm{L} / \mathrm{n}_{\mathrm{o}}, \quad \mathrm{L}^{\prime}=\mathrm{L} \operatorname{tg} \mathrm{a}_{\mathrm{e}}$

\section{Application}

The augmented matrix of the optical component in the optical path can be written in this way: If the component is only changing the direction of light and is independent of polarization, the transformation submatrix of light path should be placed at the upper left corner of the 4 elements, other positions on the main diagonal are 1 , and the remaining elements are 0 . If the component is related to polarization, while the light is still propagating in the original direction, the Jones matrix can be placed at the lower right corner of the 4 elements, other positions on the main diagonal are 1 , and the remaining elements are 0 . If the polarization device changes in the direction of light at the same time, the corresponding augmented matrix can be written according to the analysis of parallel beam splitter.

We can multiply matrix continuously through this form. However, parallel beam splitter will make different changes for the horizontal polarization component and the vertical polarization component. Therefore, in the calculation of complex optical path, we should proceed from right to left one by one, and calculate in different matrix form according to the polarization component in horizontal or vertical direction.

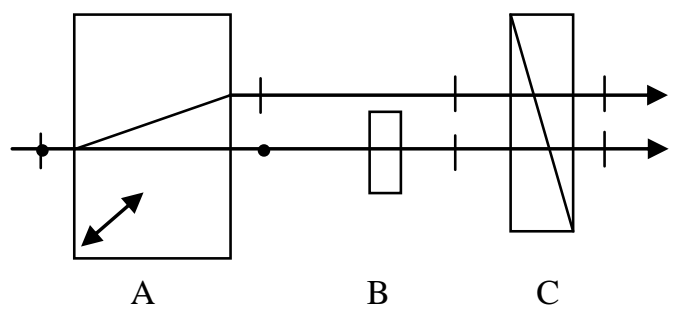

Figure 2. The structure of high efficiency polarizer. A: Parallel beam splitter, B: half wave plate, C:polarizer

High efficiency polarizer is a special device which is widely used in many field. The schematic diagram is shown in Fig 2. Next, we will use this new matrix method to analyze it. The high efficiency polarizer uses the parallel beam splitter A to bias the natural light and split the beam, and then let one of the beams pass through the half wave plate $B$. The angle between the optical axis of the half wave plate and the direction of the vibration of the beam is equal to $\pi / 4$, so that the vibration direction of the outgoing light is perpendicular to the original direction, so that the vibration direction of the two polarized light beams coming out from the parallel beam splitter is in the same direction. In order to maintain a high extinction ratio of the two beams of polarized light, a polarizing mirror $\mathrm{C}$ can be added to the optical path to increase the degree of polarization. In addition, the beam combiner should be added in actual application (it's not plotted here), which does not affect the analysis of this paper.

As shown in Fig. 2, the beam splitter $\mathrm{C}$, for the vibrating component in the direction parallel to the paper, the block diagonal matrix is 


$$
M_{P}=\left(\begin{array}{ccccc}
1 & L^{\prime} & 0 & 0 & 0 \\
0 & 1 & 0 & 0 & 0 \\
0 & 0 & 1 & 0 & 0 \\
0 & 0 & 0 & 0 & 0 \\
0 & 0 & 0 & 0 & 1
\end{array}\right)
$$

Where, $L^{\prime}=\mathrm{L} / \mathrm{ne}^{\prime}$. In the same way, the augmented matrix of half wave plate can be written

$$
M_{B}=\left(\begin{array}{lllll}
1 & 0 & 0 & 0 & 0 \\
0 & 1 & 0 & 0 & 0 \\
0 & 0 & 1 & 0 & 0 \\
0 & 0 & 0 & 0 & 1 \\
0 & 0 & 0 & 1 & 0
\end{array}\right)
$$

When the light is incident perpendicularly, for the polarized component in the direction parallel to the paper (the e light in parallel beam splitter, the top one of the light path shown in Fig 2.), we have

$$
M_{1}=M_{P} M_{\|}=\left(\begin{array}{ccccc}
1 & L_{C}^{\prime} & L^{\prime} & 0 & 0 \\
0 & 1 & 0 & 0 & 0 \\
0 & 0 & 1 & 0 & 0 \\
0 & 0 & 0 & 0 & 0 \\
0 & 0 & 0 & 0 & 1
\end{array}\right)
$$

When the light is incident perpendicularly, for the polarized component with direction perpendicular to the paper (the o light in parallel beam splitter, the lower one of the light path shown in Fig 2.), we have

$$
M_{2}=M_{P} M_{B} M_{\|}=\left(\begin{array}{ccccc}
1 & L_{C}^{\prime}+L_{A}^{\prime} & 0 & 0 & 0 \\
0 & 1 & 0 & 0 & 0 \\
0 & 0 & 1 & 0 & 0 \\
0 & 0 & 0 & 0 & 0 \\
0 & 0 & 0 & 1 & 0
\end{array}\right)
$$

Where $L_{A}^{\prime}=L_{A} / n_{o}, L_{C}^{\prime}=L_{C} / n_{e}^{\prime}$ respectively represent the optical thickness of o light and $\mathrm{e}$ light. When the incident light parameter is $A=\left(\begin{array}{llllll}r_{1} & \theta_{1} & 1 & x_{1} & y_{1}\end{array}\right)^{T}=\left(\begin{array}{lllll}r & 0 & 1 & 1 & 1\end{array}\right)^{T}$, the parallel component of emergent light is $A_{\|}=\left(\begin{array}{lllll}r_{2} & \theta_{2} & 1 & x_{2} & y_{2}\end{array}\right)^{T}=\left(\begin{array}{lllll}r & 0 & 1 & 0 & 1\end{array}\right)^{T}$, the perpendicular component of emergent light is $A_{\perp}=\left(\begin{array}{lllll}r_{2} & \theta_{2} & 1 & x_{2} & y_{2}\end{array}\right)^{T}=\left(\begin{array}{llllll}r+L^{\prime} & 0 & 1 & 0 & 1\end{array}\right)^{T}$.

As can be seen from the calculation results, the vibration direction and the propagation direction of the two outgoing light beams are the same. Only there is a displacement L' of the e light (in the parallel beam splitter), whose vibration component is parallel to the paper. When the orientation of the optical axis is 45 degrees to the incident crystal surface and the incident light is incident perpendicularly, if the thickness of the parallel beam splitter is small and the shear difference of the two beams is very small, it can be approximately regarded as a beam of light. The displacement is only in direct proportion to the thickness of the parallel beam splitter, if the orientation of the optical axis is 45 degrees to the incident crystal surface and the incident light is incident perpendicularly. The outgoing light can be approximately regarded as one beam of light. But if the two beams are too close, the wave plate will not be able to insert in one of them. We usually add a beam combiner at the back to fit the two beams together. The beam combiner greatest advantage is to reuse half of the light energy of the general 
polarized light, so that the transmittance of the original prism is doubled! This is a very special advantage and has been well applied in the design of passive components for optical communications.

\section{Summary}

Combining the transform matrix of optical elements with Jones matrix, it can describe the position and direction of light in optical path, and describe the polarization state of light. The physical meaning is clear, and the operation process is simple, and the results are clear and intuitive. But for different optical components, the augmented matrix form is not unique. It should be added or simplified according to the actual situation, and its use conditions should also be specified and explained.

\section{Acknowledgement}

This work is supported by national natural science fund project (No. 11447200); Shandong provincial natural science foundation (No.ZR201702200400); Science and technology program of Shandong higher education institutions (No. J17KA087); The program of independent innovation and achievement transformation plan for Zaozhuang (No. 2016GH19); Science and technology program of Zaozhuang (No. 2016GX31).

\section{References}

[1] S.N. Savenkov, Jones and Mueller matrices: structure, symmetry relations and information content. (2009)71-119, .

[2] L. Jinjun, L. Xiangyang, Y. Qiao, S. Xueping, G. Xiaochuan, Comparison and analysis on Polarization aberration in Pehan prisms and the Telescope system including Pehan prisms, Physics Procedia, 19(2011) 436-441.

[3] T.J. Moore, M.R. Jones, D.R. Tree, D.D. Allred, An inexpensive high-temperature optical fiber thermometer, Journal of Quantitative Spectroscopy and Radiative Transfer. 187(2017)358-363.

[4] A. Yariv, P. Yeh, Optical waves in crystals, WILEY, 1984.

[5] D. Goldstein, Polarized Light, Third Edition, Crc Press, 2010.

[6] S.N. Savenkov, Jones and Mueller matrices: structure, symmetry relations and information content, Light Scattering Reviews.4(2009)71-119.

[7] S.S. Ivanov, A.A. Rangelov, N.V. Vitanov, T. Peters, T. Halfmann, Highly efficient broadband conversion of light polarization by composite retarders, Journal of the Optical Society of America :A Optics Image Science \& Vision.29(2012)265-269.

[8] R.C. Jones, A New Calculus for the Treatment of Optical SystemsI. Description and Discussion of the Calculus, J. Opt. Soc. Am. 31(1941)488-493. 